\title{
La cromatografía de gases acoplada a espectrometría de masas como herramienta de alta selectividad para caracterizar fósiles químicos en el petróleo
}

\author{
Elena E. Stashenko*, Jairo René Martínez \\ Centro de Cromatografía y Espectrometría de Masas - CROM-MASS, Centro de Investigación de Excelencia - CENIVAM, \\ Escuela de Química, Universidad Industrial de Santander, Carrera 27, Calle 9, Bucaramanga, Colombia \\ e-mail:elena@tucan.uis.edu.co
}

\section{Resumen}

La determinación de fósiles químicos (biomarcadores) en el petróleo constituye un reto analítico formidable, porque requiere la detección y la cuantificación de moléculas específicas, presentes en cantidades a nivel de trazas, en mezclas de hidrocarburos altamente complejas. En este artículo, se resaltan algunos resultados de importancia para la geoquímica orgánica, que han sido posibles gracias al uso de la espectrometría de masas como sistema de detección, altamente sensible y selectivo, en conjunto con la alta resolución que alcanzan la cromatografía de gases monodimensional y la cromatografía de gases completa $(\mathrm{GC} \times \mathrm{GC})$. Se han seleccionado ejemplos representativos de estudios recientes de biomarcadores en la geoquímica ambiental y en la geoquímica del petróleo.

Palavras- chave

Cromatografía de gases; GC-MS; espectrometría de masas tándem; SIM; MRM; biomarcadores.

\section{Gas chromatography - mass spectrometry as a highly selective tool to characterize chemical fossils in petroleum}

\section{Abstract}

Petroleum molecular fossil (biomarker) determination constitutes a formidable analytical challenge, because it demands detecting and quantifying specific trace-level molecules within highly complex hydrocarbon mixtures. This article highlights certain results of importance to organic geochemistry, which have been possible thanks to the use of mass spectrometry as a highly sensitive and selective detection system, in conjunction with the high resolution provided by one-dimensional or comprehensive $(\mathrm{GC} \times \mathrm{GC})$ gas chromatography. Representative examples of recent biomarker studies in petroleum and environmental geochemistry have been selected.

Keywords

Gas chromatography; GC-MS; tandem mass spectrometry; SIM; MRM; biomarkers. 


\section{Introducción}

En la formación del petróleo, como resultado del aumento de temperatura y presión durante el enterramiento de material vegetal y animal, las biomoléculas sufren transformaciones químicas diversas por degradación microbiana, polimerización y/o condensación. El resultado general es una mezcla de sustancias de alto peso molecular que, en conjunto, se denomina querógeno. $\mathrm{Su}$ fracción soluble en solventes orgánicos se conoce como bitumen y constituye, eventualmente, la fuente de petróleo y gas natural ${ }^{[1]}$. En estudios realizados en la década de los 60 sobre las transformaciones de las biomoléculas hacia la mezcla, que se conoce como petróleo, se buscaron relaciones moleculares "precursor - producto" ${ }^{[2]}$. Se encontraron varios tipos de moléculas, llamadas "fósiles químicos", marcadores biológicos o biomarcadores, que poseen una relación bastante estrecha con sus precursores biológicos, por tener una estructura molecular casi inalterada, $o$ con diferencias, que pueden ser explicadas por ciertos mecanismos químicos, y que, a su vez, proporcionan información sobre diversos procesos geológicos ${ }^{[3]}$. Según su naturaleza química, dentro de los biomarcadores reportados en la literatura científica, se distinguen alcanos lineales y ramificados, isoprenoides, sesquiterpenos, diterpenoides, terpanos tricíclicos y tetracíclicos, esteranos, hidrocarburos aromáticos y porfirinas, entre otros ${ }^{[4]}$.

Una clase importante de fósiles moleculares la conforman los biomarcadores terpenoides, que comparten al isopreno $\left(\mathrm{C}_{5}\right)$, como unidad estructural, y cuyas rutas biosintéticas poseen características afectadas por eventos evolutivos, tales como la presencia o no de oxígeno molecular. Múltiples investigaciones han establecido que los hidrocarburos triterpanos pentacíclicos, con 27 - 35 átomos de carbono, son los remanentes de polioles de estructuras moleculares presentes en bacterias (hopanos) ${ }^{[5]}$. Los esteranos provienen de los esteroles de eucariotas ${ }^{[6]}$. Este campo de investigación ha sido bastante activo, y hoy se dispone de una caracterización detallada de muchos biomarcadores, su conexión con las moléculas precursoras y la relación entre los eventos geológicos y las transformaciones químicas observadas ${ }^{[7]}$. The Biomarker Guide, un libro publicado por Peters y Moldowan en $1993 \mathrm{y}$ del cual hay una $2^{\mathrm{a}}$ edición, es el libro de referencia principal en este campo, pues contiene tanto la descripción estructural de los biomarcadores, como los métodos para su análisis y la información de carácter geoquímico que portan ${ }^{[8]}$.

Las ciencias de separación han jugado un papel fundamental en las investigaciones y las aplicaciones sobre biomarcadores del petróleo, ya que proveen la capacidad para identificar y cuantificar componentes minoritarios (biomarcadores) en mezclas sumamente complejas (petróleo y sus fracciones). La principal herramienta analítica ha sido la cromatografía de gases acoplada a espectrometría de masas. Para muchas aplicaciones de clasificación de crudos o sus fracciones o comparaciones entre crudos, es posible alcanzar resultados satisfactorios con la cromatografía de gases monodimensional, apoyada en la selectividad, que se logra con la técnica de monitoreo de ion(es) seleccionado(s), SIM (por sus siglas en inglés, Selected Ion Monitoring). Sin embargo, estudios más detallados requieren niveles superiores de resolución y especificidad. En unos casos, se ha recurrido a la cromatografía completa y a la espectrometría de masas de alta resolución. En otros casos, se ha aprovechado la alta especificidad, que proporcionan algunos modos de operación de espectrómetros tándem, por ejemplo, el triple cuadrupolo (QqQ). Los artículos de revisión de este tema, escritos por Simoneit ${ }^{[9]}$ y por Medeiros y Simoneit ${ }^{[10]}$, ofrecen una visión amplia de los tipos de biomarca- 
dores y las técnicas cromatográficas usadas para su caracterización. En las siguientes secciones, se presentan ejemplos recientes de aplicaciones de las técnicas GC-MS, GCxGC-MS/TOF y GC-MS/MS, para determinar biomarcadores en el petróleo o en sus fracciones.

\section{La información selectiva de la técnica GC-MS/SIM}

Aunque gran parte de las investigaciones sobre biomarcadores han sido motivadas por su relevancia para la exploración del petróleo, su uso se ha extendido a la geoquímica orgánica en general, y se ha encontrado un campo importante de aplicación en estudios de geoquímica ambiental. La identificación de biomarcadores y su cuantificación por técnicas GC-MS permiten calcular cocientes entre biomarcadores, que han mostrado ser bastante estables, $y$ se han usado en estudios de impacto ambiental para distinguir entre diferentes fuentes de hidrocarburos. Además, el análisis isotópico permite distinguir ente orígenes antropogénico y geogénico de los hidrocarburos hallados. La utilidad de estos cocientes se aprecia en el trabajo de Fernández y colaboradores ${ }^{[11]}$, quienes mostraron que el uso de 1 cociente entre biomarcadores sesquiterpanos tricíclicos, más 3 cocientes entre hidrocarburos poliaromáticos, permitió clasificar correctamente 34 muestras de petróleo, de orígenes variados, entre 5 grupos, relacionados con su región de extracción: los crudos del Mar del Norte, los de Nigeria, los de África del Norte, los del Medio Oriente y los de otros orígenes. El análisis químico detallado de la composición muestra que, para un mismo campo petrolífero, existen diferencias entre crudos de pozos cercanos o del mismo pozo, pero extraídos de profundidades diferentes. En cambio, los cocientes entre biomarcadores son parámetros que varían menos, pero contienen, sin embargo, características distintivas del crudo, que permiten identificar su origen. Este uso de los biomarcadores, para investigar fuentes de hidrocarburos, se ilustra en el trabajo de Scholz-Böttcher y colaboradores $^{[12]}$, quienes, al identificar y cuantificar biomarcadores, pudieron distinguir 3 grupos de hidrocarburos contaminantes, según sus fuentes, en sedimentos superficiales recogidos en la costa del sur del Golfo de México. Además de hidrocarburos constituyentes de combustibles fósiles comerciales, se hallaron distribuciones de biomarcadores de una filtración asfáltica conocida en la zona, superpuestas con biomarcadores resultantes de perforaciones realizadas en depósitos del Cretácico al Terciario. La capacidad de distinguir estos 3 orígenes entre la mezcla compleja de hidrocarburos hallada, descansa en la posibilidad de diferenciar las señales correspondientes a los biomarcadores entre una gran cantidad de señales cromatográficas. Esto es posible con la técnica GC-MS, por una parte, gracias al uso de columnas cromatográficas capilares, que permiten una resolución cromatográfica alta $y$, por otra parte, por la operación del espectrómetro de masas en el modo de adquisición SIM ${ }^{[13]}$, que constituye un filtro altamente selectivo de los iones característicos, formados en la cámara de ionización.

El petróleo y sus fracciones son mezclas complejas en cuyos perfiles cromatográficos, normalmente, hay regiones denominadas UCM (por sus siglas en inglés, Unresolved Complex Mixture), que involucran una cantidad grande de picos cromatográficos no resueltos (coelución). La región, que se presenta expandida en la Figura 1, contiene un perfil cromatográfico obtenido con la técnica GC-MS en modo de barrido completo (full scan) del intervalo de masas entre $m / z 40$ y 400 , para la fracción de saturados del extracto de una muestra de esquistos de la 

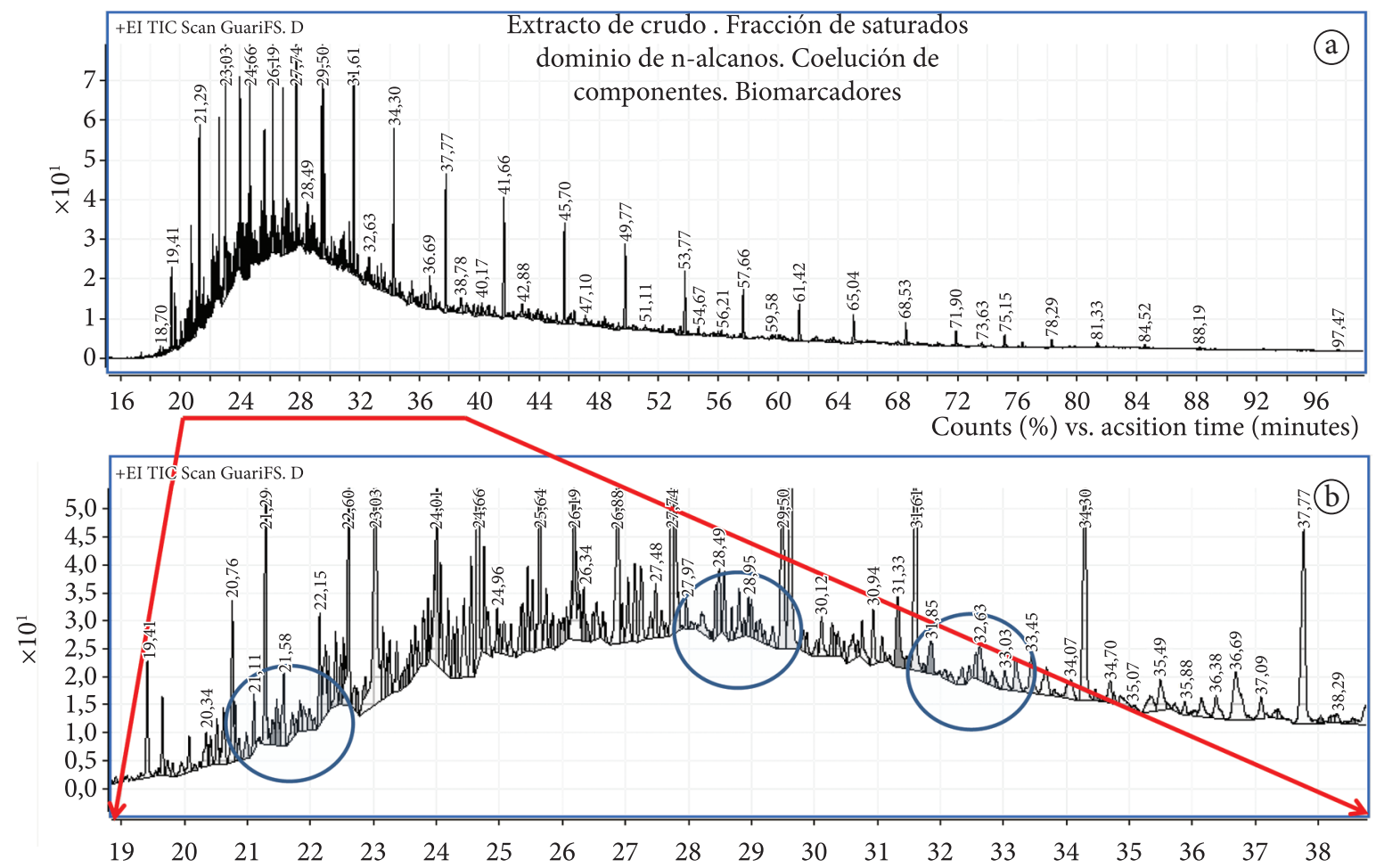

Figura 1 A. Perfil cromatográfico de la fracción de saturados de una muestra de crudo aislada de esquistos de la formación La Luna en Girón (Colombia). B. Amplificación $t_{R}=19-38$ minutos. Columna DB5-MS $(60 \mathrm{~m} \times 0.25 \mathrm{~mm} \times 0.25 \mu \mathrm{m})$. Splitless $\left(\mathrm{V}_{\text {iny. }} 2 \mu \mathrm{L}\right)$. GC-MS/MS (QqQ). Modo de adquisición: barrido completo (full scan).

formación La Luna en Girón (Departamento de Santander, Colombia). En este perfil, se aprecian múltiples superposiciones de la cantidad grande de componentes coeluidos, presentes en esta muestra compleja. Entre este conjunto complicado de señales, representados principalmente por alcanos, se encuentran los picos cromatográficos correspondientes a los fósiles moleculares, estructuras, que sufrieron pocas transformaciones y guardan una similitud grande con las biomoléculas originales.

En la Figura 2, se presentan las estructuras distintivas de hopanos, esteranos y diasteranos, 3 tipos de biomarcadores muy utilizados en la geoquímica orgánica. En cada estructura se indican sus fragmentos distintivos, característicos o "diagnósticos", que se aprovechan en la técnica GC-MS/SIM, para obtener fragmentogramas de masas para cada tipo de biomarcador. En la Figura 3, aparecen los fragmentogramas de masas de hopanos y esteranos, obtenidos al utilizar, como filtros, las señales de iones en $\mathrm{m} / z 191$ y 217, respectivamente, durante la adquisición en modo SIM, para la misma fracción de saturados del extracto de esquistos de la formación La Luna. En comparación con el perfil cromatográfico, obtenido en modo de barrido completo (Figura 1), estos fragmentogramas constituyen una "simplificación" considerable, que permite cuantificar biomarcadores individuales, o conjuntos completos de biomarcadores, que compartan un mismo fragmento "diagnóstico" molecular.

La selección de señales (i.e., iones característicos), que se logra con la técnica GC-MS/ SIM para biomarcadores, que poseen el mismo fragmento estructural, ha posibilitado muchos estudios. En numerosas aplicaciones, cuyo pro- 
pósito central es diferenciar las fracciones o los petróleos de diferentes orígenes, o sometidos a diferentes procesos de modificación de su composición, basta con disponer de esta información clasificada, aunque no se haya logrado una sepa-

Esteranos

Diasteranos
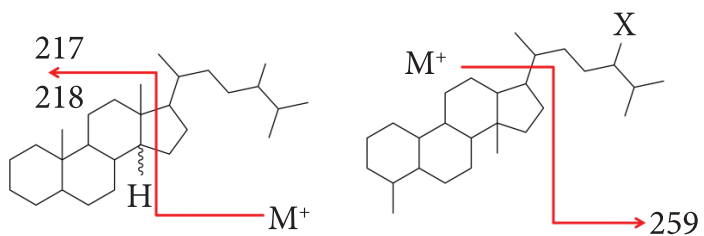

Hopanos

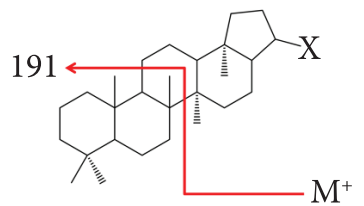

Figura 2 Fragmentos moleculares representativos (iones "diagnóstico") de 3 tipos de biomarcadores, de uso muy amplio en la caracterización del petróleo. ración completa. Para estos casos, los biomarcadores, gracias a la información depurada por la técnica GC-MS/SIM, han desempeñado un papel central, como elementos constituyentes de la "huella digital" en diferentes mezclas de hidrocarburos.

En el estudio de la contaminación ambiental, por derrames de petróleo, el uso de los biomarcadores como elementos diferenciadores ya se encuentra involucrado en normas técnicas. Por ejemplo, la norma ASTM D5739-06 se titula "Práctica estándar para la identificación de la fuente de derrame de petróleo por cromatografía de gases y espectrometría de masas de baja resolución de iones positivos e impacto de electrones". Aunque existen normas europeas y norteamericanas al respecto, el tema de la identificación de fuentes de petróleo para establecer
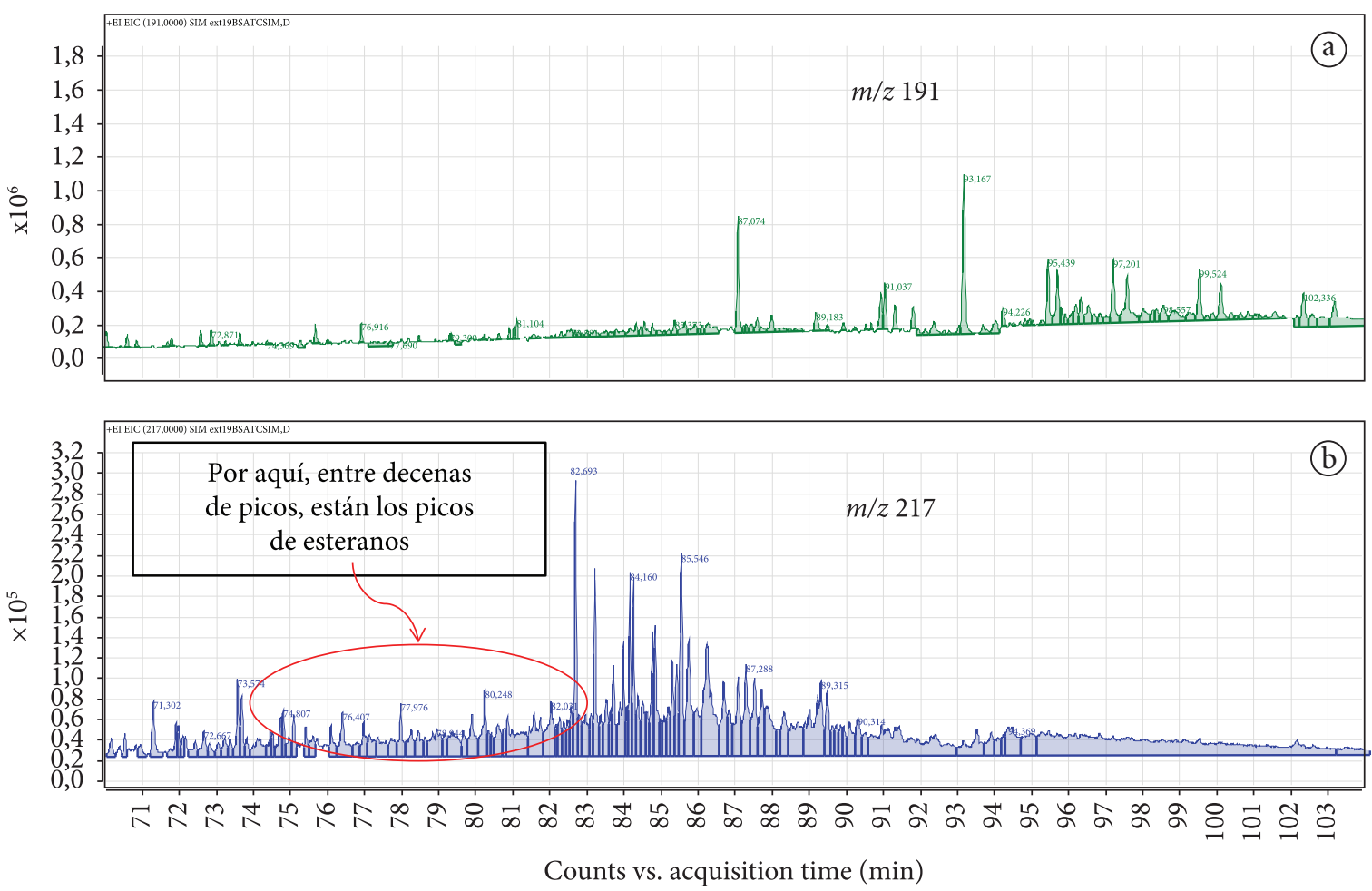

Figura 3 Fragmentogramas de masas para: A. Hopanos (m/z 191) y B. Esteranos (B, m/z 217), presentes en la fracción de saturados de un extracto de esquistos de la formación La Luna en Girón (Colombia). Columna DB5MS (60 m $\times 0.25 \mathrm{~mm} \times 0,25 \mu \mathrm{m})$. Splitless $\left(\mathrm{V}_{\text {iny. }} 2 \mu \mathrm{L}\right)$. GC-MS/MS (QqQ). Modo de adquisición: SIM. 
"huellas digitales", que permitan caracterizar crudos, continúa siendo objeto de investigación bastante activa.

Empero, persisten discusiones sobre lo subjetivas que puedan ser las comparaciones de los perfiles cromatográficos, $y$, para facilitar la toma de decisiones, se han propuesto algunos procedimientos complementarios ${ }^{[14]}$. Christensen $y$ colaboradores ${ }^{[15]}$ utilizaron el método de análisis de componentes principales (PCA) para detectar grupos de crudos similares a partir de datos cromatográficos pre-procesados. Los fragmentogramas de masas para el ion en $m / z 217$ (que selecciona las señales de esteranos tri- y tetracíclicos), fueron los datos originales y su procesamiento previo consistió en alinear los cromatogramas para corregir los desplazamientos de los tiempos de retención, causados por diferencias instrumentales. Aunque se utilizó la técnica GC-MS/ SIM, para obtener un perfil simplificado, correspondiente a un grupo de biomarcadores, el método no requirió la identificación y la cuantificación de biomarcadores específicos. En la norma ASTM D5739-06, la información básica se obtiene por GC-MS con operación en modo de monitoreo de iones seleccionados, SIM. Se especifican los valores $\mathrm{m} / z$ de los iones, que se monitorearán para 56 biomarcadores particulares, dentro de los cuales se incluyen terpanos tricíclicos, hopanos, norhopanos, esteranos, diasteranos, colestanos, ergostanos y estigmastanos. Todos estos son hidrocarburos, que poseen entre 19 y 29 átomos de carbono, que forman estructuras bastante similares de penta- $y$ hexaciclos condensados, con pequeñas diferencias en el tipo y la posición de los sustituyentes, cuyos picos cromatográficos deben estar resueltos sobre la línea base, para que su cuantificación sea más confiable. Para cumplir esta condición, durante la preparación de la muestra, se hace una separación en una columna de adsorción de gel de sílice activada, por la que se pasa la fracción de la muestra soluble en hexano y diclorometano. Los biomarcadores, a los que se hace el seguimiento según esta norma, se encuentran en la fracción de saturados (obtenida al eluir con hexano), que se somete a análisis por GC-MS/SIM. El programa de temperatura de la columna se ajusta para lograr la separación deseada de los picos de los hidrocarburos saturados, hasta la línea base $^{[16]}$.

Las columnas cromatográficas capilares, que se emplean en la caracterización de biomarcadores, son de fase apolar (polidimetilsiloxanos, fenilpolimetilsiloxanos), por ser esa la naturaleza general de los biomarcadores. Sin embargo, al aumentar los campos de aplicación y la diversidad de biomarcadores de interés, es mayor el número de situaciones en las que se requiere el uso de la fase polar. Tal es el caso de la determinación de oleanano y lupano. El oleanano es un triterpenoide pentacíclico, cuya presencia se interpreta como indicadora del carácter no-marino del ambiente de formación del querógeno y como marcador de la contribución de plantas superiores terrestres, angiospermas. El oleanano ayuda a elucidar la edad geológica del querógeno. El lupano también es un triterpano pentacíclico, derivado probablemente de angiospermas. No hay muchos reportes de su presencia en el petróleo, pero, según hallaron Nytoft y colaboradores $^{[17]}$, la causa puede haber sido que su pico cromatográfico no se resuelve, cuando se utiliza una columna con fase estacionaria apolar, y se asigna equivocadamente como un oleanano. Estos investigadores mostraron que al usar una fase estacionaria polar se logra la separación del lupano, en cuyo espectro de masas aparece un fragmento en $m / z 369$, que está ausente en el espectro del oleanano. El análisis de muestras de diferentes orígenes sugirió que el lupano está asociado con muestras provenientes de latitudes 
altas (Groenlandia, Canadá) y se constituye, por lo tanto, en otro elemento para la caracterización de crudos.

\section{La información isotópica de los biomarcadores}

En las últimas dos décadas se ha desarrollado un nuevo elemento que permite diferenciar, útil en el estudio de orígenes y edades del petróleo, basado en el fraccionamiento isotópico, que puede suceder durante la fotosíntesis, la fijación o la asimilación de moléculas pequeñas como dióxido de carbono o metano, por parte de plantas o microrganismos. En contraste con procesos físicos como la disolución de $\mathrm{CO}_{2}$ en agua, efectos cinéticos de rotura y formación de enlaces dan lugar a que las moléculas orgánicas, que sufren procesos de degradación biológica y química, posean una disminución relativa del isótopo ${ }^{13} \mathrm{C}$. El parámetro a supervisar es $\delta^{13} \mathrm{C}$, el cociente entre los contenidos de ${ }^{13} \mathrm{C} \mathrm{y}^{12} \mathrm{C}$ de una molécula, en relación con el cociente determinado para una sustancia patrón, según la fórmula 1, en la que el resultado se expresa como décimas de por cien, o por mil (\%o $)^{[18]}$. El metano atmosférico tiene un valor $\delta^{13} \mathrm{C}=-47$, mientras que los crudos de petróleo tienen valores $\delta^{13} \mathrm{C}$ entre -15 y -40 .

$$
\delta^{13} C=\left[\frac{\left(\frac{{ }^{13} C}{{ }^{12} C}\right)_{\text {muestra }}}{\left(\frac{{ }^{13} C}{{ }^{12} C}\right)_{\text {estándar }}}-1\right] 1000
$$

Para el caso del petróleo, es posible que sus componentes provengan de fuentes separadas, cada uno con diferente historia de formación y degradación. El valor $\delta^{13} \mathrm{C}$, que se determina, representa un valor compuesto. A fin de distinguir estas contribuciones, se requiere determinar las composiciones isotópicas de los constituyentes individuales. Es aquí, cuando las ciencias de separación entran a jugar un papel importante.

En la técnica GC-IRMS (por sus siglas en inglés, Isotope Ratio Mass Spectrometry) el final de la columna cromatográfica se conecta con una cámara, que contiene óxido de cobre $(\mathrm{CuO})$ o lechos metálicos tales como $\mathrm{Cu} / \mathrm{Ni} / \mathrm{Pt}$, y se calienta a $800-900{ }^{\circ} \mathrm{C}$, para lograr la combustión completa de la materia orgánica. La corriente gaseosa entra al espectrómetro de masas, que selecciona únicamente las señales en $m / z$ 44 y 45 , lo que permite calcular el cociente $\delta^{13} \mathrm{C}$ entre las cantidades $\mathrm{de}^{13} \mathrm{C} \mathrm{y}^{12} \mathrm{C}$, para la muestra y una sustancia de referencia ${ }^{[19]}$. Un ejemplo de la resolución adicional, que se logra con el análisis isotópico, es la comparación realizada por Cortés y colaboradores ${ }^{[20]}$ de crudos de los Llanos Orientales (Colombia). Un total de 65 muestras de crudos de 5 yacimientos (Apiay, Castilla, Chichimene, Cupiagua y Cusiana), se sometieron a análisis por GC-MS y GC-IRMS, para obtener cocientes de biomarcadores y valores de $\delta^{13} \mathrm{C}$ de compuestos individuales. Todas las muestras se distinguieron por su contenido relativamente alto de oleanano, que indica un carácter no-marino del ambiente de formación del querógeno; sin embargo, este dato aislado no coincidía con lo que sugerían otros cocientes de biomarcadores. Esta fue una indicación de la no-homogeneidad del origen geológico de los crudos. La comparación de los intervalos de valores de $\delta^{13} \mathrm{C}$ de los crudos mostró que, aunque todos provienen del Cretáceo, los campos de Cusiana y Cupiagua tuvieron contribuciones de rocas fuente del Terciario. Mientras los valores de $\delta^{13} \mathrm{C}$ de los crudos de los campos Apiay, Castilla y Chichimene estuvieron entre $-24,22$ y $-32,99$, los de Cusiana y Cupiagua, entre $-26,01$ y $-32,72$. Los valores de $\delta^{13} \mathrm{C}$ correspondientes a $n$-alcanos, confirmaron el carácter marino de 
la materia orgánica de los crudos de Cusiana, Cupiagua y Apiay, mientras que en los casos de Castilla y Chichimene el carácter fue marino, pero con aportes terrestres.

\section{El aumento de la resolución cromatográfica con la cromatografía completa, GC x GC}

En algunas investigaciones la resolución que se alcanza con la técnica GC-MS/SIM, no es suficiente para detectar diferencias relativamente pequeñas en la composición de crudos o sus fracciones. Por ejemplo, en estudios de biodegradación de crudos, o cuando se comparan muestras tomadas a diferentes profundidades en el mismo pozo o en pozos colindantes, los perfiles cromatográficos (barrido completo, full scan) o los fragmentogramas de masas (SIM), son casi indistinguibles o presentan problemas de interpretación debido a la superposición de picos cromatográficos (coelución). En estas situaciones, se han logrado avances considerables gracias al uso de la cromatografía completa (Comprehensive chromatography, GC $\times$ GC), en la cual se utilizan 2 columnas cromatográficas con fases estacionarias ortogonales, i.e., apolar y polar o viceversa. El flujo de la primera columna es fraccionado en múltiples segmentos, que se transfieren consecutivamente a la segunda columna (cromatografía rápida), que está conectada a un detector de alta velocidad de respuesta, normalmente, un espectrómetro de masas con analizador de tiempo de vuelo (MS-TOF) ${ }^{[21]}$. El resultado es un perfil cromatográfico bidimensional cuyos ejes son los tiempos de retención en cada columna. En la segunda dimensión, se logran resolver muchos analitos, que se superponían en la primera columna (cromatografía monodimensional).
La gran capacidad de resolución de la cromatografía completa queda demostrada en el trabajo de Tran y colaboradores ${ }^{[22]}$. Estos autores estudiaron muestras de petróleo de diferentes estados de biodegradación, así como muestras de crudos australianos de edades y procedencias diferentes. Con la resolución cromatográfica tan alta, usando las columnas en la secuencia polar-apolar, lograron resolver en muestras biodegradadas la zona UCM, que en cromatografía monodimensional aparece como un gran pico ancho. Encontraron que sus constituyentes son alifáticos, principalmente, decahidronaftalenos con sustituyentes alquílicos. La longitud de los sustituyentes alquílicos fue corta $\left(\mathrm{C}_{1}-\mathrm{C}_{3}\right)$ en muestras con poca biodegradación y mayor $\left(\mathrm{C}_{4}-\mathrm{C}_{7}\right)$, en las muestras con alto nivel de biodegradación. En un estudio similar de las UCM, que aparecen en los perfiles cromatográficos de muestras provenientes del sur de la provincia de Ontario en Canadá, Ventura y colaboradores ${ }^{[23]}$ detectaron cierta estructura o estratificación, que se manifiesta en el intervalo de tiempos de retención, cubiertos por la UCM. La identificación de sus constituyentes mostró, que aquellas UCM, que aparecen con tiempos de retención de $n$-alcanos con 15 a 20 átomos de carbono, están formadas principalmente por isómeros de compuestos policíclicos sustituidos y sin sustituyentes y contienen hasta 6 anillos. Las UCM, que aparecen con tiempos de retención posteriores, están formadas por isoprenoides diastereómeros arquéicos mono-, bi- y tricíclicos, de 36 a 40 átomos de carbono.

Otra situación, cuando se requiere resolución cromatográfica alta es la indagación de posible conectividad entre pozos de extracción de petróleo. Si existe comunicación en el subsuelo, la composición de los crudos debe ser la misma. Sin embargo, debido a la coelución, la comparación detallada de 2 crudos no es posible, 
cuando se usa cromatografía monodimensional. Para demostrar el excelente desempeño de la cromatografía completa en este tipo de situaciones, Ventura y colaboradores ${ }^{[24]}$ realizaron el análisis GC $\times$ GC-MS/TOF de 4 muestras de crudo. Dos muestras se tomaron del mismo pozo, a la misma profundidad, y las otras 2 , se recogieron en 2 pozos diferentes, interconectados por una frontera sedimentaria porosa. Gracias a que en el perfil cromatográfico bidimensional los picos cromatográficos aparecen organizados por clases de sustancias, se pudo lograr claramente, sin ambigüedades, la comparación detallada de las 4 composiciones, según estas clases o familias de compuestos. Se confirmó la concordancia en la composición dentro de las 2 parejas de muestras y se hallaron las diferencias entre las 2 parejas, según el contenido de varias clases de sustancias.

Otro resultado importante en el estudio de biomarcadores, basado en la gran capacidad de resolución de la cromatografía completa, fue la comparación de crudos de origen lacustre y marino, hecha por Oliveira y colaboradores ${ }^{[25]}$. Los biomarcadores 3b-metilhopano y onocerano se hallaron solamente en muestras de crudo de origen lacustre. En las muestras de crudo marino hubo predominancia de 2a-metilhopano, y no se encontró onocerano. Así, este conjunto de moléculas, distinguibles y fácilmente caracterizables por la cromatografía completa, GC $\times$ GC-MS/TOF, permiten diferenciar las fuentes de la materia orgánica. Este mismo grupo de investigación utilizó la cromatografía completa para caracterizar biomarcadores en gasóleo extrapesado $^{[26]}$. Además de terpanos y esteranos, se pudieron detectar hopenos y moretenos y se resolvieron terpanos tricíclicos y pentacíclicos, que típicamente coeluyen. Una manifestación de la mayor resolución de la cromatografía completa fue la identificación de un 64\% más de ter- panos tri- y tetracíclicos en comparación con lo que se había logrado para la misma muestra con la técnica GC-MS/SIM.

La aplicación de la técnica GC x GC-MS/ TOF al análisis de biomarcadores en crudos de Brasil permitió detectar, por primera vez, terpanos tri- $y$ tetracíclicos desmetilados, que en investigaciones, que usaban cromatografía monodimensional, no se habían podido resolver. Similarmente, se "eliminaron" coeluciones encontradas previamente, tales como las de hopanos y esteranos en el fragmentograma de masas basado en el registro del ion en $\mathrm{m} / z 217$, o entre terpanos tri- y pentacíclicos, en el fragmentograma con $m / z 191^{[27]}$.

Eiserbeck y colaboradores ${ }^{[28]}$ realizaron un estudio comparativo de los resultados de la caracterización de biomarcadores al emplear técnicas diferentes, i.e., GC-MS, GC × GC y GC-MS/MS, en extractos de rocas fuente del Ártico y del sudeste asiático y en crudos del Terciario y terrígenos. Los cocientes de biomarcadores homohopanos tuvieron diferencias entre el 2 y el 10\%, según la técnica empleada. La gran ventaja de disponer de la alta resolución de la cromatografía completa es que se puede analizar la muestra de crudo simplemente disuelta en diclorometano, sin fraccionamiento previo. Esto elimina la incertidumbre sobre la posible transformación química, que puedan sufrir algunos componentes del crudo (triterpenoides insaturados), al estar expuestos al campo electrostático interno de zeolitas, que se emplean como tamices moleculares durante el fraccionamiento. Otra ventaja de la técnica GC $\times$ GC-MS/TOF, que se resaltó en esta comparación, fue su capacidad de separar sustancias que poseen la misma masa molecular, pero patrones de fragmentación similares (lo que impide su distinción por SIM). 
Sin embargo, en aplicaciones, en las que se buscan familias de biomarcadores o biomarcadores específicos, la técnica más apropiada es el monitoreo de múltiples reacciones, MRM (por sus siglas en inglés, Multiple Reaction Monitoring), que se caracteriza por su especificidad muy elevada ${ }^{[13]}$.

\section{Alta especificidad con GC-MS/MS}

En la Figura 4, se presenta un esquema de la operación del triple cuadrupolo (QqQ) en el modo de monitoreo de múltiples reacciones, MRM. Los iones seleccionados, con una masa particular (por ejemplo, iones moleculares, $\mathrm{M}^{+\star}$ ), que emergen de un primer cuadrupolo, operado en modo SIM, pasan a una cámara de colisiones activadas donde padecen activación y posterior disociación. Los iones producto, que emergen de la celda de colisiones activadas, pasan a otro cuadrupolo, operado en modo SIM. El grado de fragmentación de los iones seleccionados en el primer cuadrupolo, $\mathrm{Q}_{1}$, depende, en primer lugar, de su estructura, de la naturaleza del gas de colisión $\left(\mathrm{N}_{2}, \mathrm{Ar}, \mathrm{Xe}\right)$, de la presión residual en la celda de colisiones y del voltaje, $\mathrm{V}_{\mathrm{a}}$. El voltaje acelerador, $\mathrm{V}_{\mathrm{a}}$, se aplica para aumentar la energía cinética de los iones, que se convierte en la energía interna, cuyo aumento conduce a la respectiva fragmentación $\left(\mathrm{eV}_{\mathrm{a}}=\mathrm{mv}^{2} / 2 ; \mathrm{m} \mathrm{y} \mathrm{v}\right.$ - masa $\mathrm{y}$ velocidad del ion; $\mathrm{V}_{\mathrm{a}}$ - voltaje aplicado a la celda). El primer cuadrupolo, $\mathrm{Q}_{1}$, filtra iones precursores $\left(F_{1}\right)$ y el segundo, $Q_{2}$, iones-producto $\left(F_{2}\right)$, correspondientes a una reacción de transición
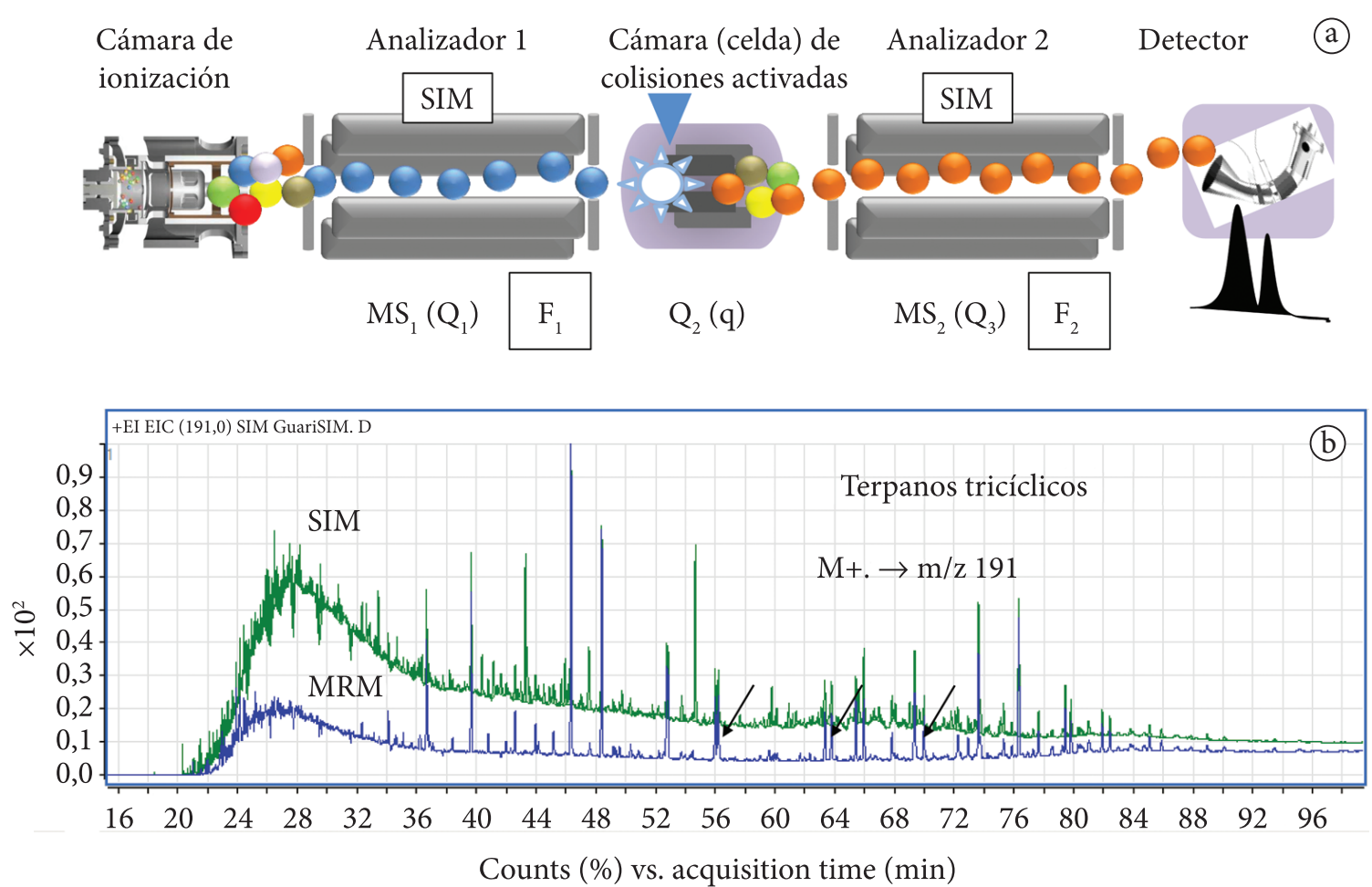

Figura 4 A. Diagrama del espectrómetro de masas de triple cuadrupolo, MS/QqQ, operado en el modo de monitoreo de múltiples reacciones, MRM. B. Comparación del fragmentograma de masas para m/z 191, con el perfil MRM para un conjunto de transiciones de iones moleculares de terpanos tricíclicos, $M^{+} \rightarrow m / z 191$, cuyo producto es el ion con $\mathrm{m} / \mathrm{z} 191$. 
$\mathrm{F}_{1} \rightarrow \mathrm{F}_{2}$, a menudo llamada, transición metaestable (Figura 4a). Debe existir la relación "genética" (parentesco) entre ambos iones y, estos dos deben ser estables, verbigracia, en el espectro de masas, obtenido en el modo full scan, exhibir señales intensas. El monitoreo, a la vez, de ambos iones, v.gr., precursor y producto, permite aumentar la especificidad del análisis. La coincidencia de una (o dos) reacción(es) de transición entre los iones, precursor y producto, de un analito-target con la misma reacción de transición de una impureza, interferencia, contaminación o la señal de background, es prácticamente imposible. Esto evita los falsos positivos y los falsos negativos en el análisis y lo hace más sensible debido al aumento de la selectividad.

El sistema GC-MS/MS (QqQ) en el modo MRM actúa como un sistema de detección cromatográfica altamente especifico. La Figura 4b presenta la comparación del fragmentograma de masas para los iones $\mathrm{m} / z 191$ de la fracción de saturados del extracto de esquistos de la formación La Luna (Girón, Colombia), con el perfil obtenido por MRM, para un conjunto discreto de transiciones (iones precursores, que son iones moleculares de una serie homóloga de hopanos), que tienen como producto el ion con $\mathrm{m} / z 191$ (Figura 2). Se aprecia una sustancial mejora de la relación señal/ruido, junto con la eliminación de muchas señales, que estaban presentes en el fragmentograma de masas, pero que no hacían parte de las transiciones monitoreadas. Esta especificidad se aprecia en la Figura 5, que muestra los perfiles cromatográficos para triterpanos tricíclicos, que difieren en 1 y 2 grupos metilénicos $(\Delta m=14$ y 28$)$. Para estos casos, los datos adqui-
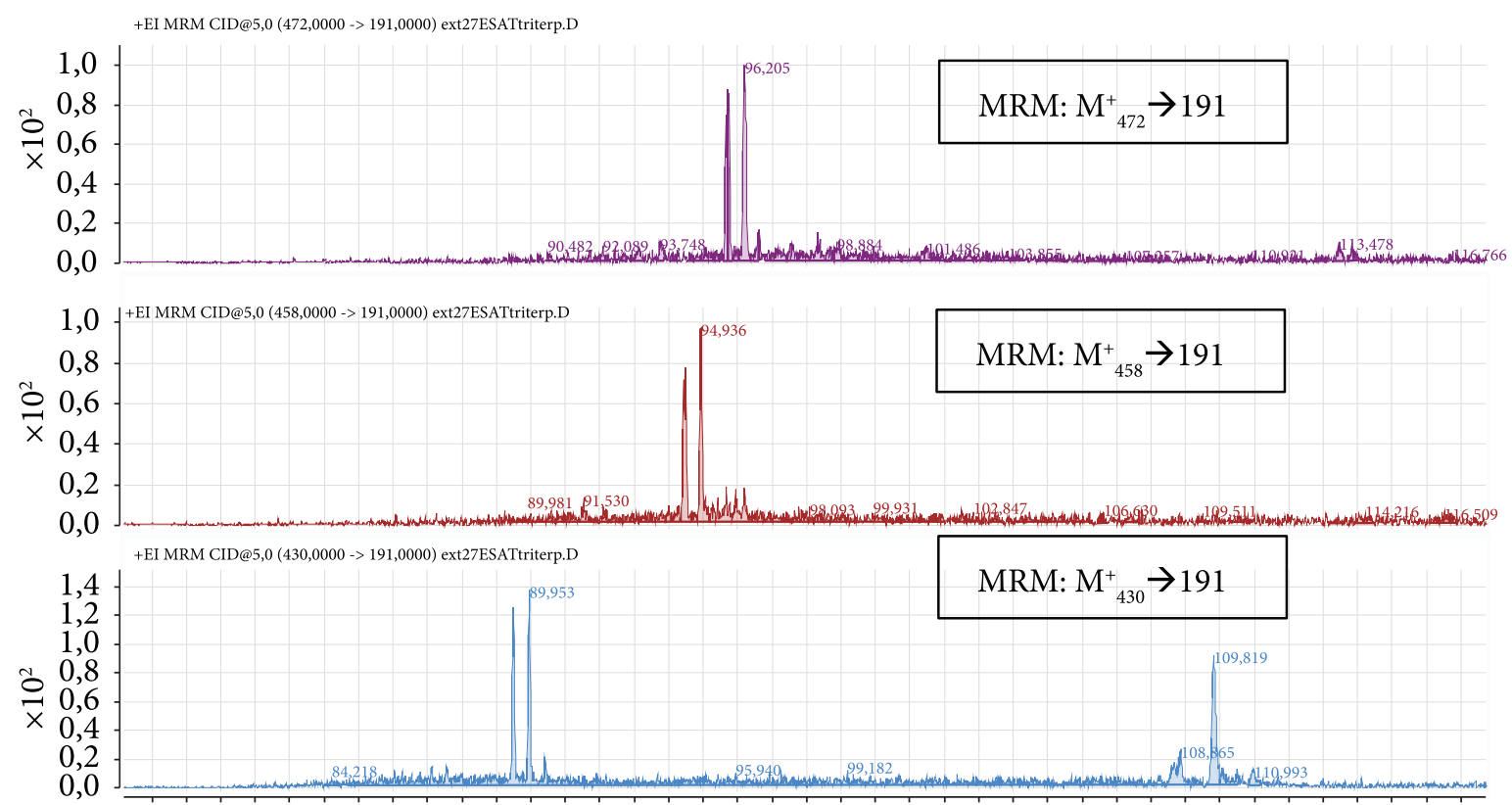

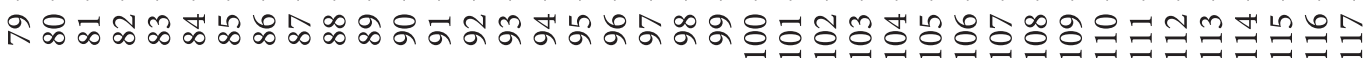

Counts vs, acquisition time ( $\mathrm{min}$ )

Figura 5 Perfiles cromatográficos obtenidos por MRM para terpanos tricíclicos, que difieren en 1 y 2 grupos metilénicos, explorados con la transición $\mathrm{M}^{+} \rightarrow \mathrm{m} / \mathrm{z}$ 191. Columna DB5-MS (60 m $\left.\times 0,25 \mathrm{~mm} \times 0,25 \mu \mathrm{m}\right)$. Splitless $\left(\mathrm{V}_{\text {iny. }} 2 \mu \mathrm{L}\right)$. GC-MS/MS (QqQ). Modo de adquisición: MRM. 
ridos en el experimento MRM, se han procesado para mostrar solamente una transción a la vez $\left(\mathrm{M}_{472}^{+} \rightarrow 191 ; \mathrm{M}_{458}^{+} \rightarrow 191 ; \mathrm{M}^{+}{ }_{430} \rightarrow 191\right)$.

En la caracterización geoquímica de crudos se relaciona el cociente entre los isómeros del metildibenzotiofeno con la madurez de la roca fuente. Sin embargo, debido a la coelución, el cálculo de este cociente está sujeto a errores, incluso cuando se emplean fragmentogramas de masas. Chiaberge y colaboradores ${ }^{[29]}$ desarrollaron un método para el cálculo de este cociente con mayor confiabilidad, por medio de GC-MS/ MS, operado en modo MRM. El método fue tan específico para los dibenzotiofenos, que no fue necesario fraccionar previamente la muestra de crudo. En otra aplicación, Liang y colaboradores $^{[30]}$ pudieron cuantificar confiablemente los diamantoides en una muestra de crudo, cuya preparación consistió simplemente en su dilución en un solvente orgánico. Esto se logró luego de optimizar la energía en la celda de colisiones, los tiempos de retención y los iones precursores y producto, que se utilizaron en el modo MRM, para lograr la selectividad y la sensibilidad máximas para los diamantoides, que fueron superiores a las alcanzadas con la técnica GC-MS/SIM.

\section{Conclusiones}

En la técnica de acoplamiento de cromatografía de gases con espectrometría de masas, la espectrometría de masas ha jugado un papel fundamental en el aprovechamiento de la información que se desprende de la detección y cuantificación de los fósiles moleculares, en aplicaciones diversas de la geoquímica orgánica. El acoplamiento GC-MS permite combinar sinérgicamente la alta resolución cromatográfica con las sensibilidades y selectividades altas de la espectrometría de masas, para detectar cantidades traza de biomarcadores dentro de mezclas de hidrocarburos altamente complejas. En los casos, cuando persisten las coeluciones, a pesar de la alta selectividad del método SIM, se puede recurrir a la gran capacidad de resolución de la cromatografía completa o a la alta especificidad de las técnicas de la espectrometría de masas tándem, en particular, el modo de adquisición MRM.

\section{Agradecimientos}

Los autores agradecen la financiación de Colciencias y la Agencia Nacional de Hidrocarburos (ANH) (Contrato ANHColciencias RC-747-2009).

\section{Referencias}

1 Speight J. The chemistry and technology of petroleum. Marcel Dekker, Inc. New York, 1991, 760 p.

2 Streibl M, Herout V. Terpenoids-especially oxygenated mono-, sesqui-, di-, and triterpenoids. In: Eglinton G, Murphy MTJ, editors. Organic geochemistry: Methods and results. Berlin: Springer-Verlag; 1969. p. 402-424.

3 Eglinton G, Calvin M. Chemical fossils. Scientific American 1967; 216:32-43.

4 Demaison G, Bradley H. Genetic classification of petroleum system. AAPG Bulletin 1991; 75(10):1626-1643.

5 Taylor RF. Bacterial triterpenoids. Microbiological Reviews 1984; 48:181-198.

6 Volkman JK. A review of sterol markers for marine and terrigenous organic matter. Organic Geochemistry 1986; 9(2):83-99. http://dx.doi. org/10.1016/0146-6380(86)90089-6

7 Gaines S, Eglinton G, Rullkötter J. Echoes of life - What fossil molecules reveal about earth history. New York: Oxford University Press; 2008.

8 Peters KE, Walters CC, Moldowan JM. The Biomarker Guide. 2nd ed. New York: Cambridge University Press; 2005.

9 Simoneit B. A review of current applications of mass spectrometry for biomarker/molecular tracer elucidations. Mass Spectrometry Reviews 2005; 24:719-765. PMid:15534872. 
10 Medeiros PM, Simoneit BR. Gas chromatography coupled to mass spectrometry for analyses of organic compounds and biomarkers as tracers for geological, environmental, and forensic research. Journal of Separation Science 2007; 30:1516-1536. PMid:17623433.

11 Fernández-Varela R, Andrade JM, Muniategui S, Prada D. Selecting a reduced suite of diagnostic ratios calculated between petroleum biomarkers and polycyclic aromatic hydrocarbons to characterize a set of crude oils. Journal of Chromatography A 2010; 1217(52):8279-8289. PMid:21081235. http:// dx.doi.org/10.1016/j.chroma.2010.10.043

Scholz-Böttcher B, Ahlf S, Vázquez-Gutiérrez F, Rullkötter J. Natural vs. anthropogenic sources of hydrocarbons as revealed through biomarker analysis: A case study in the southern Gulf of Mexico. Boletín de la Sociedad Geológica Mexicana 2009; 61(1):47-56.

13 Stashenko E, Martínez J. GC-MS: Más de un analizador de masas, ¿para qué? Scientia Chromatographica. 2010;2(4):33-59.

14 Gómez-Carracedo M, Ferré J, Andrade J, Fernández-Varela R, Boqué R. Objective chemical fingerprinting of oil spills by partial least-squares discriminant analysis. Analytical and Bioanalytical Chemistry 2012; 403(7):2027-2037. http://dx.doi. org/10.1007/s00216-012-6008-5

15 Christensen J, Tomasi G, Hansen A. Chemical fingerprinting of petroleum biomarkers using time warping and PCA. Environmental Science and Technology 2005; 39:255-260. http://dx.doi. org/10.1021/es049832d

16 Wang Z, Fingas M, Li K. Fractionation of ASMB oil, identification and quantitation of aliphatic, aromatic and biomarker compounds by GC/FID and GC/MSD. Journal of Chromatographic Science 1994 32:361-366 (Parte I), 367-382 (Parte II). http://dx.doi.org/10.1093/ chromsci/32.9.361

17 Nytoft H, Bojesen-Koefoed J, Christiansen F, Fowler M. Oleanane or lupane? Reappraisal of the presence of oleanane in Cretaceous-Tertiary oils and sediments. Organic Geochemistry 2002; 33:1225-1240. http:// dx.doi.org/10.1016/S0146-6380(02)00138-9

18 Nguyen T, Bocherens H, Mariotti A, Baudin F, Pons D, Broutin $\mathrm{J}$ et al. Ecological distribution of Cenomanian terrestrial plants based on ${ }^{13} \mathrm{C} /{ }^{12} \mathrm{C}$ ratios. Palaeogeography, Palaeoclimatology,
Palaeoecology 1999; 145:79-93. http://dx.doi. org/10.1016/S0031-0182(98)00092-3

19 Hayes T, Freeman K, Popp B, Hoham C. Compound specific isotope analyses: a novel tool for reconstruction of biogeochemical processes. Organic Geochemistry 1990; 16:1115-1128. PMid:11540919. http://dx.doi.org/10.1016/0146-6380(90)90147-R

20 Cortes J, Rincon J, Jaramillo J, Philp P, Allen J. Biomarkers and compound-specific stable carbon isotope of $n$-alkanes in crude oils from Eastern Llanos Basin, Colombia. Journal of South American Earth Sciences 2010; 29;198-213.

21 Kinghorn R, Marriott P, Dawes P. Design and implementation of comprehensive gas chromatography with cryogenic modulation. Journal of Separation Science 2000; 23(3):245-252. http:// dx.doi.org/10.1016/j.jsames.2009.03.010

22 Tran T, Logan G, Grosjean E, Ryan D, Marriott P. Use of comprehensive two-dimensional gas chromatography/time-of-flight mass spectrometry for the characterization of biodegradation and unresolved complex mixtures in petroleum. Geochimica et Cosmochimica Acta 2010; 74:6468-6484. http://dx.doi. org/10.1016/j.gca.2010.08.024

23 Ventura G, Kenig F, Reddy C, Frysinger G, Nelson R, Van Mooy B et al. Analysis of unresolved complex mixtures of hydrocarbons extracted from Late Archean sediments by comprehensive two-dimensional gas chromatography (GCxGC). Organic Geochemistry 2008; 39(7):846-867. http:// dx.doi.org/10.1016/j.orggeochem.2008.03.006

24 Ventura G, Raghuraman B, Nelson R, Mullins O, Reddy C. Compound class oil fingerprinting techniques using comprehensive two-dimensional gas chromatography (GCxGC). Organic Geochemistry 2010; 41:1026-1035. http://dx.doi.org/10.1016/j.orggeochem.2010.02.014

25 Oliveira C, Ferreira A, Oliveira C, Azevedo D, Santos Neto EV, Aquino Neto FR. Biomarkers in crude oil revealed by comprehensive two-dimensional gas chromatography time-of-flight mass spectrometry: Depositional paleoenvironment proxies. Organic Geochemistry 2012; 46:154-164. http://dx.doi. org/10.1016/j.orggeochem.2012.03.002

26 Ávila B, Aguiar A, Gomes A, Azevedo D. Characterization of extraheavygas oilbiomarkers using comprehensive two-dimensional gas chromatography coupled to time-of-flight mass spectrometry. Organic 
Geochemistry 2010; 41:863-866. http://dx.doi. org/10.1016/j.orggeochem.2010.03.008

27 Aguiar A, Silva A, Azevedo D, Aquino F. Application of comprehensive two-dimensional gas chromatography coupled to time-of-flight mass spectrometry to biomarker characterization in Brazilian oils. Fuel 2010; 89:2760-2768. http://dx.doi.org/10.1016/j. fuel.2010.05.022

28 Eiserbeck C, Nelson R, Grice K, Curiale J, Reddy C. Comparison of GC-MS, GC-MRM-MS, and GC x GC to characterise higher plant biomarkers in Tertiary oils and rock extracts. Geochimica et Cosmochimica Acta 2012; 87:299-322.

29 Chiaberge S, Fiorani T, Cesti P. Methyldibenzothiophene isomer ratio in crude oils: Gas chromatography tandem mass spectrometry analysis. Fuel Processing Technology 2011; 92:21962201. http://dx.doi.org/10.1016/j.fuproc.2011.07.011

30 Liang Q, Xiong Y, Fang C, Li Y. Quantitative analysis of diamondoids in crude oils using gas chromatographytriple quadrupole mass spectrometry. Organic Geochemistry 2012; 43:83-91. http://dx.doi. org/10.1016/j.orggeochem.2011.10.008 